\title{
ORIGINAL ARTICLE $C R A F$ gene fusions in pediatric low-grade gliomas define a distinct drug response based on dimerization profiles
}

\author{
P Jain ${ }^{1,2,3}$, TM Fierst ${ }^{3,4}$, HJ Han ${ }^{1,3}$, TE Smith ${ }^{3}$, A Vakil ${ }^{3}$, PB Storm ${ }^{1,3,5,6}$, AC Resnick ${ }^{1,3,5,6,7,10}$ and AJ Waanders ${ }^{5,6,8,9,10}$
}

Pediatric low-grade gliomas (PLGGs) are commonly associated with BRAF gene fusions that aberrantly activate the mitogenactivated protein kinase (MAPK) signaling pathway. This has led to PLGG clinical trials utilizing RAF- and MAPK pathway-targeted therapeutics. Whole-genome profiling of PLGGs has also identified rare gene fusions involving another RAF isoform, CRAF/RAF1, in PLGGs and cancers occuring in adults. Whereas BRAF fusions primarily dysregulate MAPK signaling, the CRAF fusions QKI-RAF1 and SRGAP3-RAF1 aberrantly activate both the MAPK and phosphoinositide-3 kinase/mammalian target of rapamycin (PI3K/mTOR) signaling pathways. Although ATP-competitive, first-generation RAF inhibitors (vemurafenib/PLX4720, RAFi) cause paradoxical activation of the MAPK pathway in BRAF-fusion tumors, inhibition can be achieved with 'paradox breaker' RAFi, such as PLX8394. Here we report that, unlike BRAF fusions, CRAF fusions are unresponsive to both generations of RAFi, vemurafenib and PLX8394, highlighting a distinct responsiveness of CRAF fusions to clinically relevant RAFi. Whereas PLX8394 decreased BRAF-fusion dimerization, CRAF-fusion dimerization is unaffected primarily because of robust protein-protein interactions mediated by the $\mathrm{N}$-terminal non-kinase fusion partner, such as QKI. The pan-RAF dimer inhibitor, LY3009120, could suppress CRAF-fusion oncogenicity by inhibiting dimer-mediated signaling. In addition, as CRAF fusions activate both the MAPK and PI3K/mTOR signaling pathways, we identify combinatorial inhibition of the MAPK/mTOR pathway as a potential therapeutic strategy for CRAF-fusiondriven tumors. Overall, we define a mechanistic distinction between PLGG-associated BRAF- and CRAF/RAF1 fusions in response to RAFi, highlighting the importance of molecularly classifying PLGG patients for targeted therapy. Furthermore, our study uncovers an important contribution of the non-kinase fusion partner to oncogenesis and potential therapeutic strategies against PLGG-associated CRAF fusions and possibly pan-cancer CRAF fusions.

Oncogene (2017) 36, 6348-6358; doi:10.1038/onc.2017.276; published online 14 August 2017

\section{INTRODUCTION}

Pediatric low-grade gliomas (PLGGs) represent a heterogeneous group of commonly diagnosed brain tumors in children, ${ }^{1}$ with histologies ranging from pilocytic astrocytomas (PAs; WHO grade I) to diffuse fibrillary astrocytomas (WHO grade II). Alterations in the mitogen-associated protein kinase (MAPK) pathway are frequent in PLGGs, specifically KIAA1549-BRAF gene fusion in $\mathrm{PAs}^{2,3}$ and BRAF-V600E mutation mostly in Pleomorphic Xanthoastrocytomas. ${ }^{4}$ Comprehensive whole-genome sequencing studies have discovered a diversity of novel RAF-fusion gene combinations. In particular, multiple gene fusions harboring $C R A F$ (or RAF1), a serine/threonine kinase first discovered as the $v$-raf oncogene in transforming mouse sarcoma virus, ${ }^{5}$ have been reported in PLGGs. QKI-RAF1 and FYCO-RAF1 have been identified as rare alterations in PAs using whole-genome sequencing, ${ }^{6}$ whereas SRGAP3-RAF1 was first reported as a tandem duplication event. ${ }^{7,8}$ Recently, ATG7-RAF1 fusions were reported in anaplastic Pleomorphic Xanthoastrocytomas without BRAF-V600E. ${ }^{9}$ Although SRGAP3-RAF1 was shown to activate the MAPK pathway, no further studies with RAF1 fusions have been reported.
Interestingly, several adult cancers such as prostate cancer, ${ }^{10,11}$ breast cancer, ${ }^{12}$ pancreatic cancer ${ }^{13}$ and thyroid cancer ${ }^{12}$ also harbor CRAF fusions. However, the true prevalence, oncogenic mechanism and sensitivity of pan-cancer CRAF fusions to targeted therapeutics remain unknown.

The prevalence of RAF fusions in PLGGs led to studies examining the therapeutic efficacy of RAF inhibitors (RAFi). ATPcompetitive, first-generation RAFi, such as vemurafenib (research analog PLX4720), have been FDA-approved for BRAF-V600E malignant melanoma ${ }^{14}$ but were found to be ineffective in targeting BRAF fusions because of paradoxical activation of the MAPK pathway. ${ }^{3}$ Interestingly, second-generation RAFi PLX8394 could successfully target BRAF fusions, hence termed 'paradox breaker'.,15 These studies highlight the differential sensitivity of RAF mutations. While ATP-competitive RAFi inhibits wild-type BRAF and CRAF kinase activity at similar $\mathrm{IC}_{50}$ in vitro, ${ }^{15}$ the effect of RAFi on CRAF fusions versus BRAF fusions is yet to be clinically defined.

Despite recent focus on the kinase fusion partner in fusiondriven cancers, the non-kinase partner is increasingly recognized

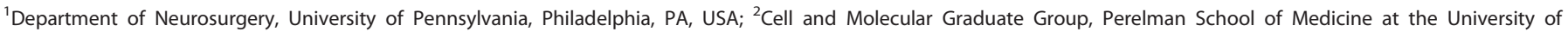

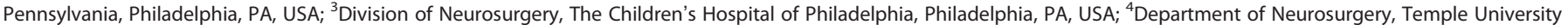

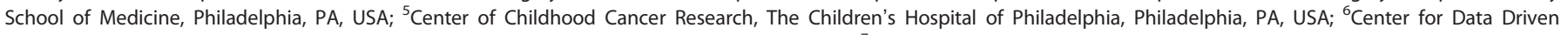

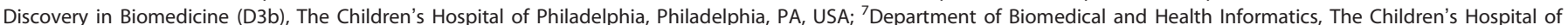

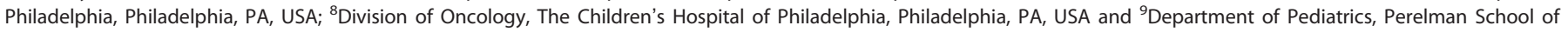

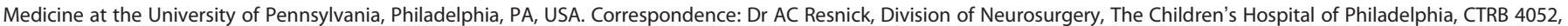

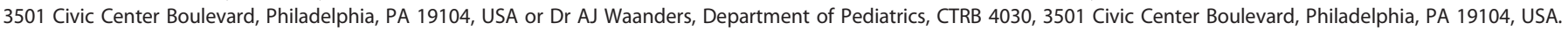
E-mail: RESNICK@email.chop.edu or WAANDERSA@email.chop.edu

${ }^{10}$ These authors are co-senior authors.

Received 15 February 2017; revised 26 June 2017; accepted 5 July 2017; published online 14 August 2017
} 
as important to oncogenesis. The $\mathrm{N}$-terminal fusion partners of CRAF fusions, such as in QKI (QKI-RAF1) and SRGAP3 (SRGAP3RAF1), have been previously implicated in cancers. QKI is an RNAbinding protein that regulates glial development and central nervous system myelination. ${ }^{16,17}$ We previously identified QKI's contribution as a fusion partner in MYB-QKI fusion in angiocentric gliomas, ${ }^{18}$ corroborating previous findings that $\mathrm{QKI}$ deletions are oncogenic in cancers such as glioblastomas, ${ }^{19,20}$ prostate cancer, ${ }^{21}$ lung cancer ${ }^{22}$ and gastric cancer. ${ }^{23}$ SRGAP3, which is a member of the SLIT-ROBO Rho-GTPase-activating protein (srGAP) family regulating actin cytoskeleton dynamics, ${ }^{24}$ has been reported as a tumor suppressor-like gene in breast cancer. ${ }^{25}$ These studies suggest the potential involvement of QKI and SRGAP3 in CRAFfusion-driven tumors.

To address these questions, we performed cellular, molecular and in vivo assays to test oncogenic mechanisms and therapeutic response of two PLGG-associated CRAF fusions, QKI-RAF1 and SRGAP3-RAF1. We found that CRAF fusions activated both the MAPK and the phosphoinositide-3 kinase/mammalian target of rapamycin (PI3K/mTOR) pathways and were unresponsive to 'paradox-breaker' RAFi. We identified robust, RAFi-resistant dimerization mediated by the non-kinase fusion partner in CRAF fusions. To overcome this RAF-directed therapeutic challenge, we characterize other potential targeting approaches for CRAF-fusiondriven tumors including the pan-RAFi LY3009120 and combinatorial targeting of the MAPK and PI3K/mTOR pathways.

\section{RESULTS}

QKI-RAF1 and SRGAP3-RAF1 are oncogenic fusions that activate the MAPK and PI3K signaling pathways

Similar to most BRAF fusions, QKI-RAF1 and SRGAP3-RAF1 lack the $\mathrm{N}$-terminal non-catalytic, inhibitory domain of RAF but retain the functional kinase domain (Figure 1a). Loss of N-terminal domains of CRAF can activate oncogenic properties, ${ }^{26,27}$ but such studies on PLGG CRAF fusions are limited to a single report on SRGAP3RAF1. ${ }^{8}$ QKI-RAF1 contains QKI exons $1-3$ encoding QKI homodimerization domain and part of its RNA-binding domain (Figure 1a). In SRGAP3-RAF1, SRGAP3 exons 1-10 encode the FesCIP4-homology domain and a coiled-coil domain (together called F-BAR domain) with dimerization properties, ${ }^{28}$ but the central Rho-GAP and C-terminal SH3 domains are lost (Figure 1a).

Although CRAF fusions are rare events in PLGGs compared with BRAF fusions, the overall prevalence in childhood cancers is unknown because of lack of genomic studies to enrich for such fusions. Nonetheless, the occurrence of diverse CRAF fusions across pediatric and adult cancers (Figure $1 \mathrm{~b}$ ) highlights the growing importance of CRAF fusions and need to understand its mechanism of oncogenicity and its sensitivity to targeted inhibition. CRAF fusions listed in Figure $1 \mathrm{~b}$ all lack the N-terminal auto-inhibitory domains of CRAF, strongly suggesting constitutive activation of CRAF kinase activity. We sought to test this hypothesis in PLGG-associated CRAF fusions, SRGAP3-RAF1 and QKI-RAF1.

The absence of patient-derived PLGG cell models has limited our understanding of the underlying biology of gene fusions. To circumvent the lack of established PLGG patient-derived CRAFfusion cell lines, we generated two heterologous cell models by stably expressing SRGAP3-RAF1 and QKI-RAF1 in Tp53-null primary mouse astrocytes (PMAs) ${ }^{6}$ and NIH3T3 (Supplementary Figures $1 \mathrm{a}$ and b). Because of the slow-growing nature of PLGGs, previous studies on PLGG-derived mutations have found the need for additional mutational hits to support transformation of primary cell lines. ${ }^{6}$ The Tp53-null background in PMAs, although alone insufficient to drive oncogenesis, ${ }^{6}$ provides a primary, glial cellular context poised for transformation and testing of gliomaassociated CRAF fusions. We also use NIH3T3s that have been predictive of oncogenic transformation and clinical response in the BRAF-fusion setting. ${ }^{29}$ To assess the oncogenic potential of CRAF fusions, we performed soft agar assays and measured colony formation of fusion-expressing cells. QKI-RAF1 and SRGAP3-RAF1 overexpression was sufficient to drive significant anchorageindependent growth of PMAs in soft agar compared with vector controls (Figure 1c, $P$-value $<0.01-0.001$ ), and we observed MAPK pathway activation by QKI-RAF1 and SRGAP3-RAF1 in PMAs (Figure 1d).

In CRAF-fusion expressing NIH3T3, we observed similar robust colony formation in soft agar assays (Figure $1 \mathrm{e}, P$-value $<0.05-$ 0.001 ). Both the MAPK and PI3K/mTOR pathways were aberrantly activated by QKI-RAF1 and SRGAP3-RAF1 as monitored by elevated phosphorylated-MEK and phospho-AKT/S6 levels in $\mathrm{NIH} 3 \mathrm{T3s}$, respectively (Figure 1f). The difference in CRAF-fusionmediated activation of both MAPK and PI3K/mTOR pathways in NIH3T3 versus only MAPK pathway in PMAs could not be explained by differential expression level of fusion proteins among cell lines (Supplementary Figure 1a) and is likely due to variation in signaling programs in different cellular contexts.

To test tumor formation in vivo, QKI-RAF1 and SRGAP3-RAF1expressing PMAs were intracranially injected into the cerebral cortex of NOD scid gamma (immunodeficient) mice. Both CRAF fusions, but not vector control-expressing PMAs, formed tumors and led to poor survival in all injected mice (Figure 1g, $P$-value $<0.01)$. Similarly, only the NIH3T3-expressing QKI-RAF1 and SRGAP3-RAF1 injected into flanks of NSG mice formed robust xenograft tumors (Figure $1 \mathrm{~h}, P$-value $<0.001$ ). Our observations in two independent cell systems suggest that QKI-RAF1 and SRGAP3RAF1 are driver oncogenes.

First- and second-generation RAFi do not suppress QKI-RAF1 and SRGAP3-RAF1

Despite clinical testing of ATP-competitive RAFi against PLGGs, no preclinical studies exist to show the effect of first- and secondgeneration RAFi (Vemurafenib/PLX4720 and PLX8394, respectively) on CRAF fusions. In QKI-RAF1 expressing NIH3T3, both PLX4720 and PLX8394 caused paradoxical activation of the MAPK pathway as seen by increasing phosphorylated-MEK and -ERK with increasing drug concentrations (Figure 2a). Interestingly, we observed decreased phosphorylated-S6 with higher RAFi despite increased phosphorylated AKT ${ }^{\text {T308 }}$ (Figure 2a), suggesting some downregulation of the PI3K pathway. Instead of suppressing growth in soft agar, both RAFi caused increased colony formation in QKI-RAF1 expressing NIH3T3 (Figure 2b).

We observed similar paradoxical activation of the MAPK pathway, PI3K/mTOR pathway suppression (Figure 2c) and lack of growth suppression in soft agar (Figure 2d) upon treating SRGAP3-RAF1 expressing NIH3T3 with PLX4720 and PLX8394. These findings distinguish CRAF fusions from BRAF-fusion (KIAA159-BRAF) that shows pathway suppression with PLX8394 (Supplementary Figure 2). This is in contrast to previous in vitro studies reporting low biochemical IC 50 for PLX8394 targeting wildtype BRAF and CRAF. ${ }^{15}$

In CRAF-fusion expressing PMAs, we observed no MAPK pathway suppression with PLX8394 treatment (Figure 2e) and no growth suppression of QKI-RAF1 (Figure 2f) and SRGAP3-RAF1 (Figure 2g) with increasing PLX4720 or PLX8394. Collectively, these findings suggest a correlation between drug-induced paradoxical MAPK pathway activation and RAFi resistance of CRAF fusions. Despite high RAFi concentrations affecting the PI3K pathway by some unknown mechanism, lack of growth inhibition in soft agar suggests that the PI3K/mTOR pathway could be secondary to MAPK pathway in CRAF-fusion expressing tumors. Neither of the tested PLX compounds affected the stability of CRAF-fusion oncoproteins in our model systems (Supplementary Figure 5a). 
a

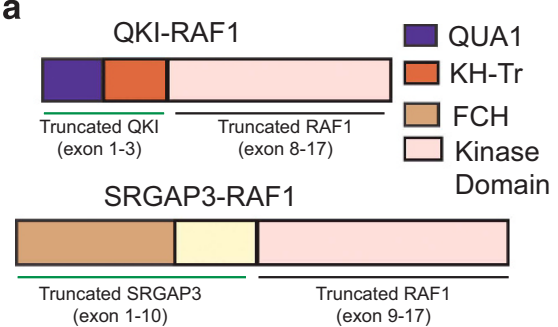

b

\begin{tabular}{|l|l|}
\hline \multicolumn{1}{|c|}{ Cancer Type } & CRAF/RAF1 fusion \\
\hline Prostate Cancer & $\begin{array}{l}\text { ESRP1-RAF1 (Ref. 10) } \\
\text { AGGF1-RAF1 (Ref. 11) }\end{array}$ \\
\hline Breast Cancer & CMTM8-RAF1 (Ref. 12) \\
\hline Pancreatic Cancer & HACL1-RAF1 (Ref. 13) \\
\hline Thyroid cancer & AGGF1-RAF1(Ref. 12) \\
\hline Pediatric low-grade glioma & FYCO1-RAF1 (Ref. 6) \\
& ATG7-RAF1 (Ref. 9) \\
\hline
\end{tabular}

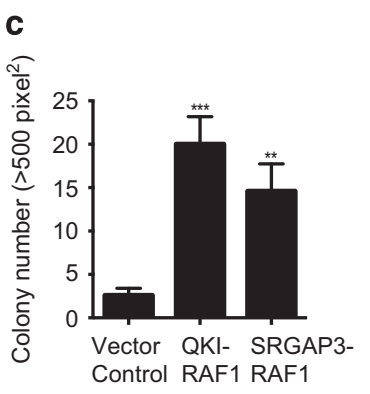

Mouse Astrocytes

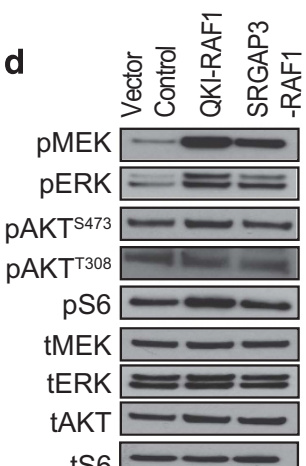

tS6

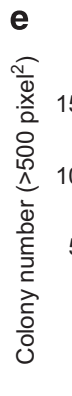

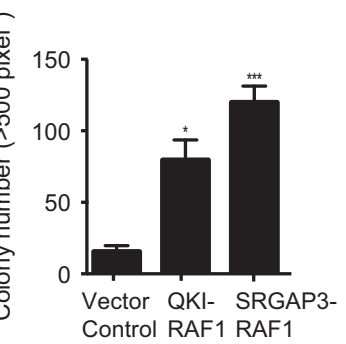

NIH3T3

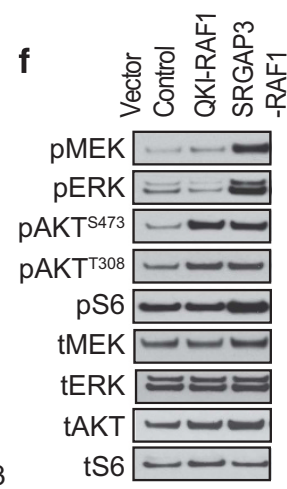

g

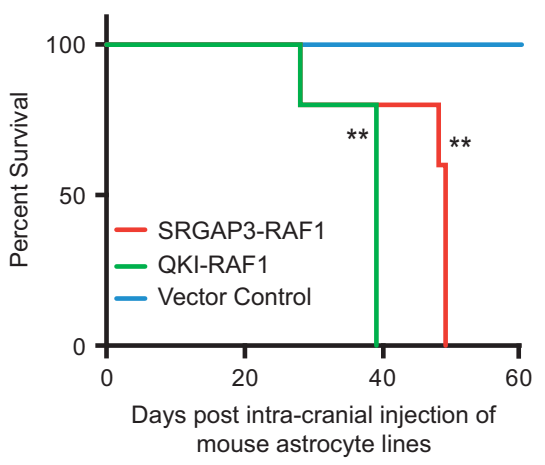

h

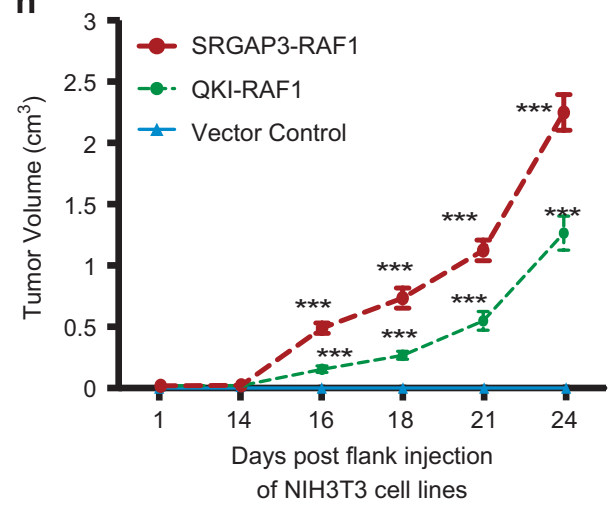

Figure 1. QKI-RAF1 and SRGAP3-RAF1 are oncogenic via activation of MAPK and PI3K pathways. (a) Structure of CRAF fusions in PLGGs. QKIRAF1: QKI exons 1-3 encode QUA1 dimerization domain and a truncated K-homology domain (KH-Tr), and CRAF/RAF1 exons 8-17 encode the protein kinase domain. SRGAP3-RAF1: SRGAP3 exons 1-10 encode the Fes/CIP4-Homology (FCH) domain and, RAF1 exons 9-17 encode CRAF kinase domain. (b) Table showing different CRAF fusions present in various adult cancers and pediatric cancer. (c, e) Soft agar assay using (c) p53-null mouse astrocyte cells (PMAs) and (e) NIH3T3 stably expressing CRAF fusions, $n=5$ and 3, respectively. Error bars represent s.e.m. (d, f) Western blot analysis of MAPK and PI3K/mTOR pathway proteins in (d) PMAs and (f) NIH3T3. (g) Kaplan-Meier survival plot of NSG mice orthotopically injected with PMAs overexpressing CRAF fusions, $n=5$ mice, $P<0.01$. (h) Flank xenograft tumor growth measurements with stable NIH3T3-expressing CRAF fusions. Error bars represent s.e.m., $n=10$ mice. ${ }^{*} P$-value $<0.05$, ${ }^{* *} P$-value $<0.01$, ${ }^{* * *} P$-value $<0.001$.

CRAF fusions exist as homodimers and heterodimers with B/CRAF/ $\mathrm{N}$-terminal fusion partner that are resistant to second-generation paradox breakers

PLX8394 prevents RAF dimerization by interacting with residues on the regulatory spine of wild-type RAF kinases, ${ }^{15}$ but our findings suggest that CRAF fusions do not respond to PLX8394 like wild-type CRAF. As dimerization underlies RAF kinase activity ${ }^{30}$ and homodimerization drives the oncogenic activity of KIAA1549$\mathrm{BRAF}^{3}$ we hypothesized that CRAF fusions are activated via homodimerization and/or heterodimerization. The presence of dimerization domains in both N-terminal QKI ${ }^{31,32} / \mathrm{SRGAP}^{28}$ and C-terminal RAF $1^{30,33}$ suggests that both partners in CRAF fusions may contribute to dimerization.

To assess the dimerization potential of CRAF fusions, we tested QKI-RAF1 homodimerization or heterodimerization with wild-type
BRAF, CRAF, QKI and truncated QKI (exons 1-3) using myc- and flag-tagged constructs in HEK293 that demonstrates high transfection efficiency. Upon immunoprecipitation (IP) of QKIRAF1 with a Myc antibody, we observed QKI-RAF1 interacting with itself, wild-type BRAF, CRAF, QKI and truncated QKI but not the vector control (Figure 3a). Similarly, we found SRGAP3-RAF1 to homodimerize and heterodimerize with wild-type BRAF and wildtype CRAF (Supplementary Figure 3a).

Next, we assessed whether PLX8394's inability to inhibit CRAF fusion is related to the inhibitor's effect on CRAF-fusion proteinprotein interactions. We found that PLX8394 failed to disrupt QKIRAF1 homodimerization and heterodimerization with wild-type B/ CRAF or QKI (Figure 3b). This is in contrast to PLX8394's disruption of KIAA1549-BRAF homodimerization (Figure 3c) and inhibition of oncogenic growth. ${ }^{3}$ This suggests that the robust correlation 

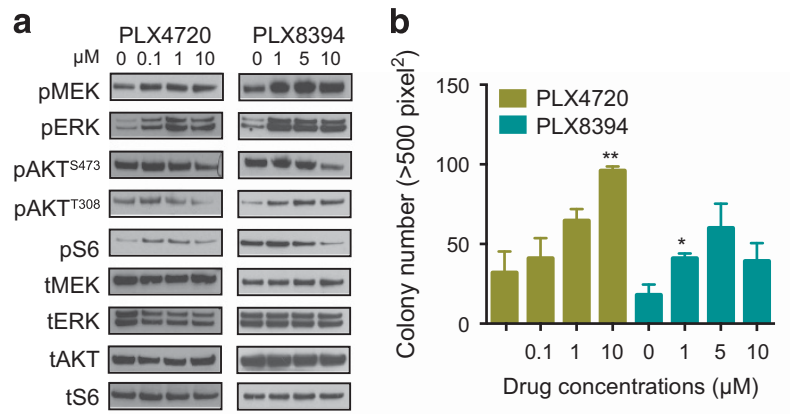

QKI-RAF1- NIH 3T3

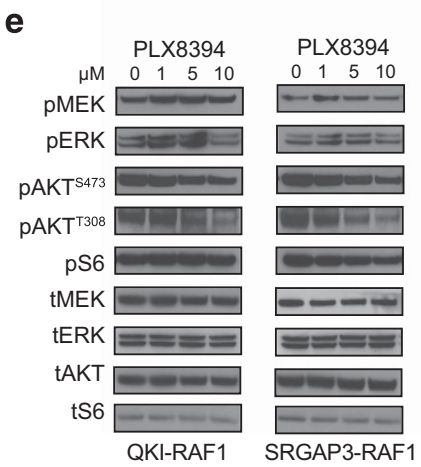

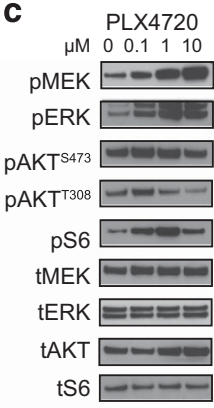

tS6
PLX8394 01510

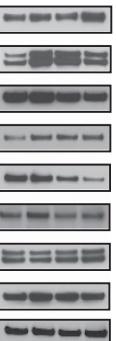

SRGAP3-RAF1- NIH 3T3
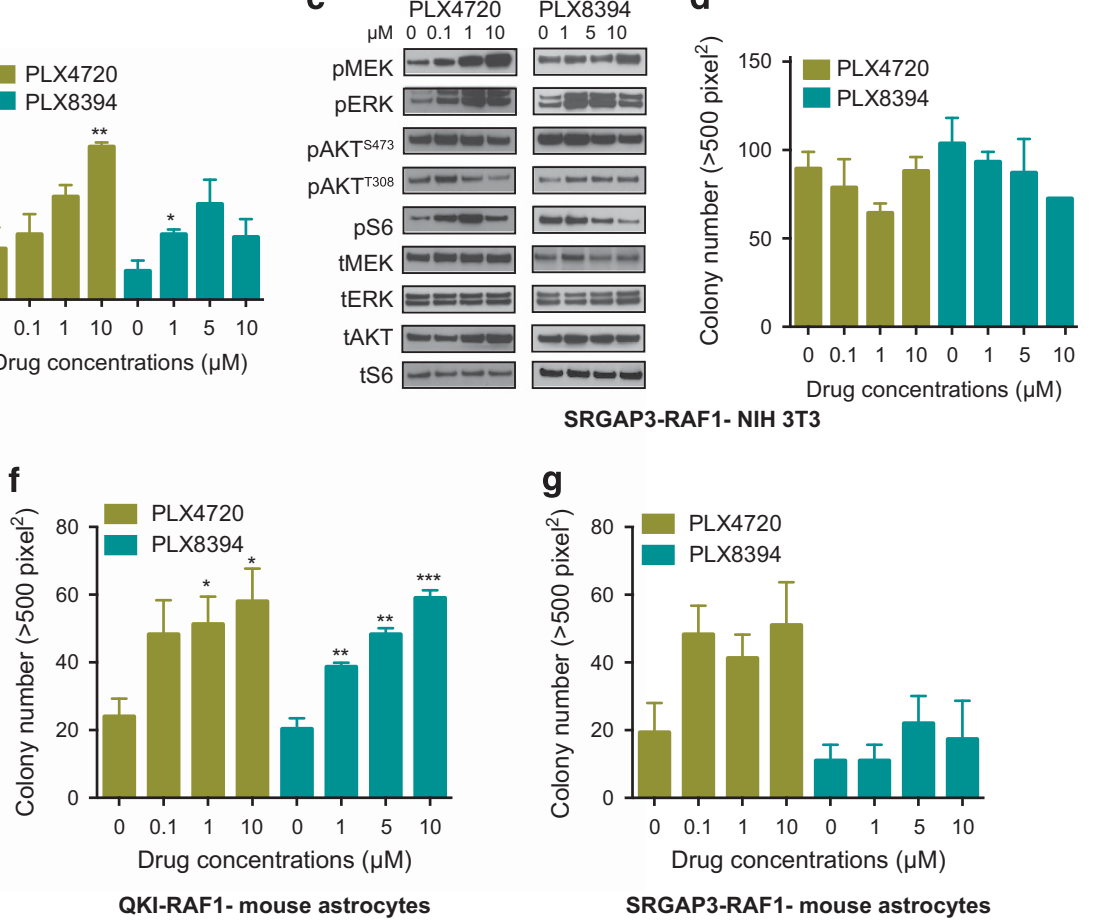

g

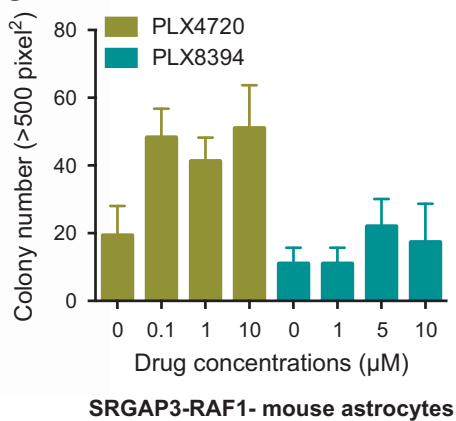

Figure 2. Existing RAF inhibitors do not suppress CRAF-fusion-driven signaling pathways and oncogenic phenotype. (a) Western blot and (b) soft agar colony analysis showing the effect of RAF inhibitors, PLX8394 and PLX4720, on MAPK and PI3K/mTOR pathways in QKI-RAF1 expressing NIH3T3. Similar (c) western blot and (d) soft agar colony analysis for treated SRGAP3-RAF1-expressing NIH3T3. (e) Western blot assay to assess effect of PLX8394 on MAPK and PI3K/mTOR pathways in CRAF-fusion expressing PMAs. (f, g) Soft agar growth of PLX8394treated PMAs expressing (f) QKI-RAF1 and (g) SRGAP3-RAF. Error bars represent s.e.m., $n=3$. No value on bars means N.S. (non-significant), ${ }^{*} P$-value $<0.05,{ }^{*} P$-value $<0.01,{ }^{* *} P$-value $<0.001$ compared with zero-drug conditions.

between dimer disruption and responsiveness to RAFi could be underlying the observed differences between BRAF- and CRAF fusions.

CRAF fusions activate oncogenic signaling in a dimerizationdependent manner

QKI-RAF1 and SRGAP3-RAF1 retain kinase activity as well as critical residues of the RAF1 dimer interface. ${ }^{30,34}$ Point mutation in a key RAF1 dimer interface residue R401H has been shown to disrupt RAF1 dimerization with little to no effect on basal kinase activity. ${ }^{35-40}$ We assessed the effect of such mutation on QKIRAF1 (QKI-RAF1 ${ }^{\text {R401H }}$ ). In co-IP assays, QKI-RAF1 ${ }^{\text {R401H }}$ homodimerization with QKI-RAF1 was unaffected (Figure 4a) but heterodimerization with wild-type BRAF and CRAF was decreased (Figures $4 \mathrm{~b}$ and $\mathrm{c}$ ), as with wild-type QKI (Figure 4d) by unknown mechanisms. Overall, the RAF1 dimer interface is critical for QKIRAF1's interaction with wild-type B/CRAF, but is only partly responsible for QKI-RAF1 homodimerization. This contrasts our previous findings with KIAA1549-BRAF where homodimerization is dependent on the BRAF dimer interface. ${ }^{3}$

The QUA1 region of $\mathrm{N}$-terminal QKI contains dimerization residues and the E48G point mutation causes disruption of QKI dimers and RNA-binding function. ${ }^{32,41}$ This mutation (QKI ${ }^{\mathrm{E} 48 \mathrm{G}}$ RAF1) decreased homodimerization with QKI-RAF1 (Figure 4a) and heterodimerization with CRAF and QKI (Figures $4 c$ and d), indicating the importance of QKI dimerization residues in QKIRAF1. Surprisingly, wild-type CRAF and $\mathrm{QKI}^{\mathrm{E} 48 \mathrm{G}}-\mathrm{RAF} 1$ interaction was also affected, even though CRAF interaction was expected to be mediated by RAF1 portion of QKI-RAF1, not QKI. One possibility is that QKI-RAF1 forms homodimers, followed by CRAF binding to form complex oligomers, so loss of QKI ${ }^{\mathrm{E} 48 \mathrm{G}}$-RAF1 homodimerization could be preventing CRAF interaction (Figure 4c), but this hypothesis needs to be tested further.
Next, we tested double point mutants of QKI and RAF1 (QKI ${ }^{\mathrm{E} 48 \mathrm{G}}$ $\left.\mathrm{RAF}^{\mathrm{R} 401 \mathrm{H}}\right)$. QKI ${ }^{\mathrm{E} 48 \mathrm{G}}-\mathrm{RAF1} 1^{\mathrm{R} 401 \mathrm{H}}$ demonstrated severe loss of interaction with QKI-RAF1, wild-type BRAF, CRAF and QKI (Figures $4 a-d)$. Taken together, these results suggest that the $\mathrm{N}$-terminal fusion partner (QKI) primarily mediates dimerization of CRAF fusion with little contribution from RAF1 dimerization motif.

We assessed the effect of dimerization mutants on QKI-RAF1's oncogenic signaling by infecting PMAs with various dimer mutant constructs and testing MAPK signaling. QKI-RAF1 ${ }^{\mathrm{R} 401 \mathrm{H}}$ drove similar levels of phosphorylated-MEK, -ERK and -S6 as QKI-RAF1, whereas $\mathrm{QKI}^{\mathrm{E} 48 \mathrm{G}}-\mathrm{RAF} 1$ and $\mathrm{QKI}^{\mathrm{E} 48 \mathrm{G}}-\mathrm{RAF}^{\mathrm{R} 401 \mathrm{H}}$ had significantly reduced MAPK signaling (Figure 4e) and $\mathrm{QKI}^{\mathrm{E} 48 \mathrm{G}}-\mathrm{RAF1}{ }^{\mathrm{R} 401 \mathrm{H}}$ showed reduced phospho-S6. These results strongly suggest that the QKI fusion partner is driving QKI-RAF1 dimerization and subsequent activation of oncogenic MAPK signaling. Soft agar assays showed a nonsignificant decrease in colony formation by QKI-RAF1 ${ }^{\text {R401H }}$ compared with QKI-RAF1 (Figure 4f, $P$-value $>0.05$ ). In contrast, QKI ${ }^{\mathrm{E} 48 \mathrm{G}}-\mathrm{RAF1}$ and $\mathrm{QKI}^{\mathrm{E} 48 \mathrm{G}}-\mathrm{RAF1}{ }^{\mathrm{R} 401 \mathrm{H}}$ displayed significant reduction in anchorage-independent growth (Figure $4 \mathrm{f}$, $P$-value $<0.01)$, suggesting that QKI-RAF1-driven oncogenic growth is largely dependent on QKI dimerization residues. For SRGAP3-RAF1, we observed decreased MAPK signaling and lower colony formation in soft agar for PMAs expressing SRGAP3RAF1 $^{\mathrm{R} 401 \mathrm{H}}$ versus SRGAP3-RAF1 (Supplementary Figures $3 \mathrm{~b}$ and c), indicating partial importance of RAF1 dimerization motif in SRGAP3-RAF1.

$\mathrm{N}$-terminal fusion partner loses functionality in CRAF fusions Loss of QKI's RNA-binding function has been detected in adult glioblastomas ${ }^{42}$ and lung cancers. ${ }^{22}$ As QKI-RAF1 retains part of QKI RNA-binding motif, we tested QKI-RAF1's RNA-binding potential compared with wild-type QKI. We performed UV 


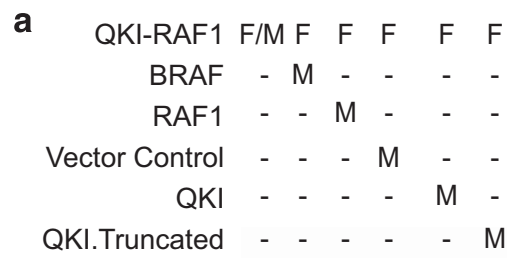
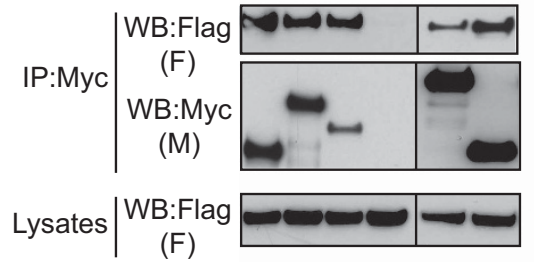

b

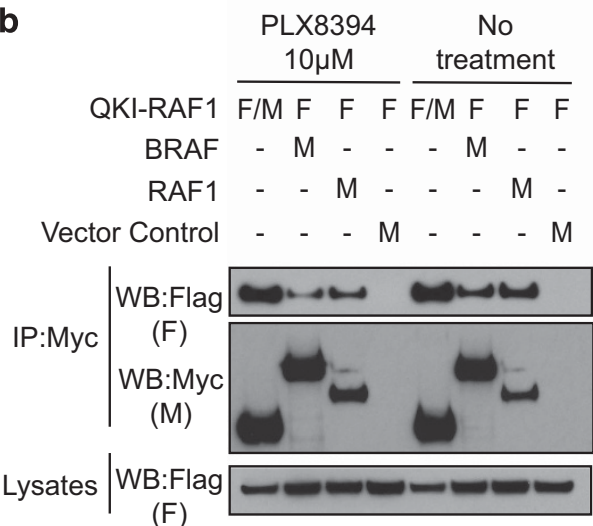

C

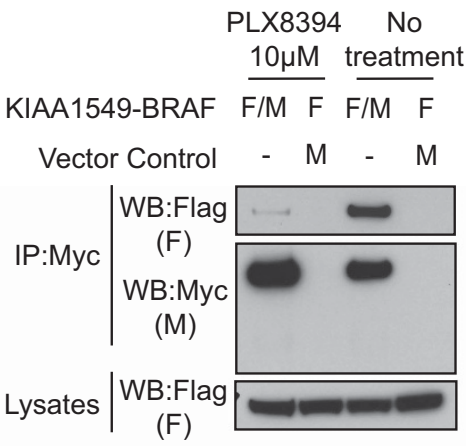

(F)

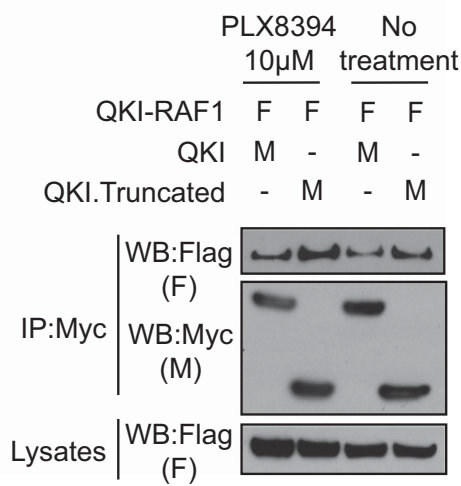

Figure 3. Dimerization profile of CRAF fusion underlies unresponsiveness to PLX8394. (a) Co-IP assay shows QKI-RAF1 dimerization with itself, and with wild-type BRAF, CRAF and QKI in HEK293 cells. (b, c) Co-IP assays to show the effect of paradox breaker/second-generation RAF inhibitor, PLX8394, on (b) QKI-RAF1 and (c) KIAA1549-BRAF dimerizations in HEK293 cells.

crosslinking by radiolabeling well-known RNA targets, myelin basic protein and early growth response gene- 2 (EGR-2) ${ }^{43}$ and incubating with vector control, QKI and QKI-RAF1 proteins expressed in HEK293 (Supplementary Figure 4a). Compared with vector control, QKI bound to both RNA targets but QKI-RAF1 failed to bind either RNA target (Figure $4 \mathrm{~g}$ ), indicating that QKI-RAF1 has lost RNA-binding function. We have previously shown that partial QKI loss in MYB-QKI expressing PLGGs causes increased oncogenicity $^{18}$ and a similar mechanism warrants more testing in QKI-RAF1 tumors.

LY3009120 inhibits CRAF fusions by inducing inactive homodimers and heterodimers with $\mathrm{B} / \mathrm{CRAF}$ and preventing QKI heterodimerization

To target the dimerization-dependent oncogenicity of CRAF fusions, we tested LY3009120, a novel RAF dimer inhibitor capable of binding and stabilizing both promoters of a RAF dimer in an inactive conformation. LY3009120 has shown efficacy in suppressing mutant BRAF dimers in melanoma, colorectal and thyroid cancer lines and circumventing the paradoxical activation caused by vemurafenib that binds only one protomer of a RAF dimer. ${ }^{44-46}$

In CRAF-fusion expressing NIH3T3s, LY3009120 led to potent, dose-dependent inhibition of both MAPK and PI3K/mTOR pathways (Figure 5a), and even low doses suppressed anchorageindependent growth (Figure $5 \mathrm{~b}, P$-value $<0.001$ ). Similar suppression of the MAPK pathway was observed with LY3009120 in CRAFfusion expressing PMAs (Figure 5c), whereas the PI3K pathway was not inhibited as strongly as in NIH3T3s. LY3009120 suppressed soft agar colony growth of PMAs (Figure 5d) verifying LY3009120 antitumor effects on CRAF fusions. The protein stability of CRAF fusions was not affected in the presence of increasing doses of LY3009120 in treated cells (Supplementary Figure 5b).

To evaluate the inhibitory mechanism of LY3009120 in CRAF fusions, we performed co-IP assays with LY3009120. Treatment with LY3009120 induced QKI-RAF1 homodimerization and QKIRAF1/BRAF heterodimerization (Figure 5e, left panel) with minimal effect on QKI-RAF1/CRAF heterodimerization. Interestingly, LY3009120 caused complete disruption of QKI-RAF1/full-length QKI heterodimerization but interaction with N-terminal-truncated QKI is retained (Figure 5e, right panel). Despite stabilizing QKIRAF1 homodimers and heterodimers with other RAF proteins, LY3009120 inhibits RAF1 kinase activity and downstream MAPK/ PI3K signaling (Figures 5a-c). LY3009120 seems to affect the QKIdimerization residues via some unknown mechanism, which could alter QKI-RAF1 homodimerization and downstream signaling. Thus, LY3009120 may provide a therapeutic alternative to existing RAFi in CRAF-fusion-driven tumors and warrants additional testing.

CRAF fusions are partially suppressed in vivo by downstream MEK inhibitors

The lack of preclinical efficacy with first- and second-generation RAFi in CRAF fusions led us to test FDA-approved MEK inhibitors (MEKi), selumetinib and trametinib. NIH3T3s overexpressing QKIRAF1 showed a dose-dependent decrease in phospho-ERK with selumetinib and trametinib, indicating on-target MEK1/2 inhibition (Figure 6a, first and middle panels, respectively) and significant growth inhibition in soft agar assays (Figure 6b, top panel, $P$-value $<0.01,0.1)$. PMAs expressing QKI-RAF1 also displayed similar pathway inhibition (Figure 6a, last panel) and 
a

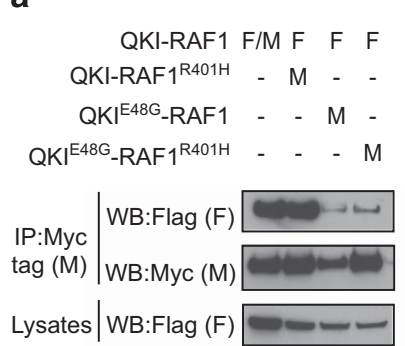

b

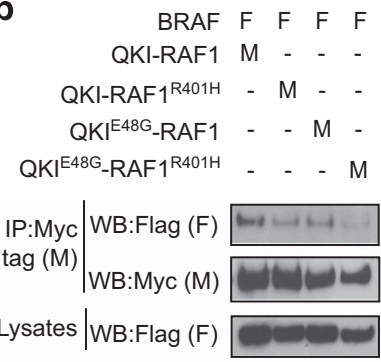

C

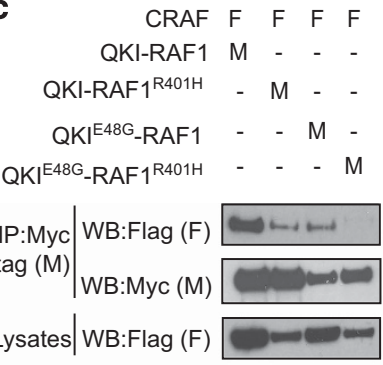

d

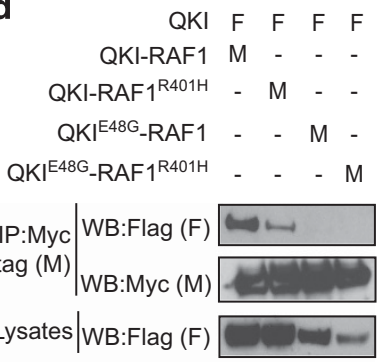

e

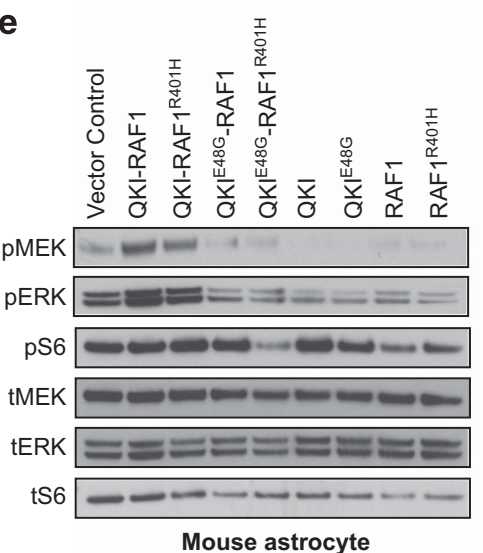

f

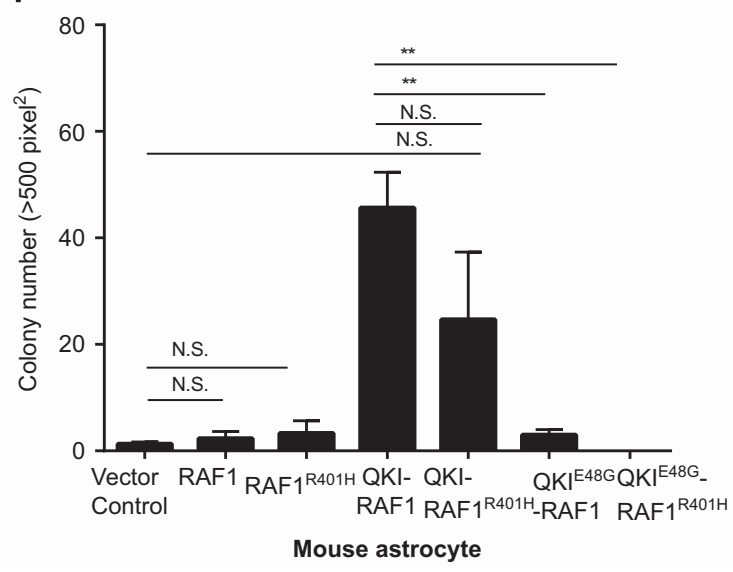

g RNA probe: $M B P$ EGR-2

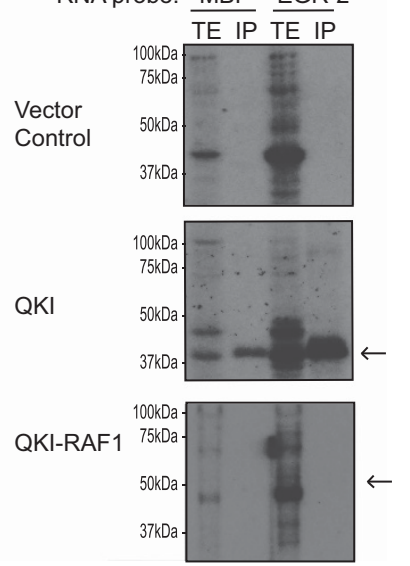

Figure 4. CRAF fusions activate oncogenic signaling in a dimerization-dependent manner. (a-d) Co-IP assays using single dimerization mutants of QKI (QKIE48G-RAF1) and RAF1 (QKI-RAF1R401H) and double dimerization mutants (QKIE48G -RAF1R401H) of QKI-RAF1 against (a) QKI-RAF1, (b) BRAF, (c) CRAF/RAF1 and (d) QKI. (e, f) PMAs expressing QKI-RAF1 mutants were assessed for (e) MAPK and PI3K pathway signaling and (f) colony formation in soft agar. (g) UV crosslinking assay with wild-type QKI and QKI-RAF1 assessing RNA binding to probes, myelin basic protein (MBP) and EGR-2, in HEK293. Error bars represent s.e.m., $n=3$. No value on bars means N.S. (non-significant), ${ }^{*} P$-value $<0.05,{ }^{* *} P$-value $<0.01,{ }^{* * *} P$-value $<0.001$ compared with vector control or QKI-RAF1 as indicated.

suppression of colony formation (Figure 6b, bottom panel, $P$-value $<0.01$ ) with trametinib.

SRGAP3-RAF1 expressing cells show similar MAPK pathway suppression with both MEKi treatments (Figure 6c). However, only trametinib, but not selumetinib, suppressed SRGAP3-RAF1mediated colony formation in NIH3T3 and PMAs (Figure 6d). These in vitro studies suggest that trametinib is more potent at inhibiting MAPK signaling and in vitro colony formation driven by CRAF fusions.

To test trametinib in vivo, we injected CRAF-fusion expressing $\mathrm{NIH} 3 \mathrm{~T} 3$ as flank xenograft tumors in NSG mice. Mice were treated oral-gavaged with trametinib but, despite significant activity in vitro, trametinib monotherapy only delayed but did not prevent QKI-RAF1 and SRGAP3-RAF1 tumor growth (Figures $6 \mathrm{e}$ and $f$, respectively). Tumor progression despite continued trametinib treatment suggests that alternative pathways activated by CRAF fusions in vivo permit tumor growth despite MEK inhibition.

Combinatorial targeting of MAPK and PI3K/mTOR pathways is more potent than single-agent therapies against CRAF fusions As the PI3K/mTOR pathway is also activated in CRAF-fusion expressing NIH3T3, we performed combinatorial targeting of both MAPK and PI3K/mTOR pathways using MEKi trametinib and mTORi everolimus, and observed decreased phospho-ERK and phosphoS6 (Figure 7a, left panel). Dose-escalation with everolimus alone failed to affect CRAF-fusion-driven signaling pathways (Supplementary Figure 4b). Despite minimal activation of baseline $\mathrm{PI} 3 \mathrm{~K} / \mathrm{mTOR}$ pathway in CRAF-fusion expressing PMAS, combinatorial treatment suppressed phospho-ERK and phospho-S6 (Figure 7a, right panel). Co-targeting inhibited anchorageindependent growth mediated by QKI-RAF1 in NIH3T3 and PMAs
(Figure 7a). Similar pathway suppression results upon co-targeting MEK and mTOR in SRGAP3-RAF1-expressing cells (Figure 7c), along with decreased colony formation in soft agar (Figure 7d). The kinase inhibitors did not destabilize CRAF-fusion proteins, even at higher doses, suggesting that trametinib and everolimus target RAF1 kinase activity but not protein stability (Supplementary Figures $5 \mathrm{c}$ and $\mathrm{d}$ ).

We next tested co-targeting in two in vivo models: NIH3T3 flank xenografts and orthotopic intracranial (IC) tumors with PMAs. Mice with QKI-RAF1 expressing NIH3T3 flank xenografts were treated with single-agent everolimus or trametinib and show initial suppression of tumor engraftment but the tumor eventually grows, whereas combinatorial treatment prolonged tumor suppression due to direct inhibition of both pathways (Figure 7e and Supplementary Figure 4c). We observed similar responsiveness in SRGAP3-RAF1-expressing NIH3T3 flank xenografts (Figure 7f). We further assessed treatment efficacy in a more relevant PLGG model, QKI-RAF1 expressing PMAs as IC tumor model. In combotreated animals, $80 \%(4 / 5)$ were alive at 50 days post-IC injection of QKI-RAF1 PMAs, whereas all vehicle and single-agent-treated animals either died or developed neurological symptoms requiring killing (Figure 7g). Our findings show that combinatorial therapy with MEKi and RADi prolonged overall survival in CRAFfusion in vivo models and should be further explored in the preclinical setting.

\section{DISCUSSION}

Our study describes the oncogenic mechanism and therapeutic sensitivity of PLGG-associated CRAF gene fusions that can greatly aid precision medicine approaches. To our knowledge, this is the 
a

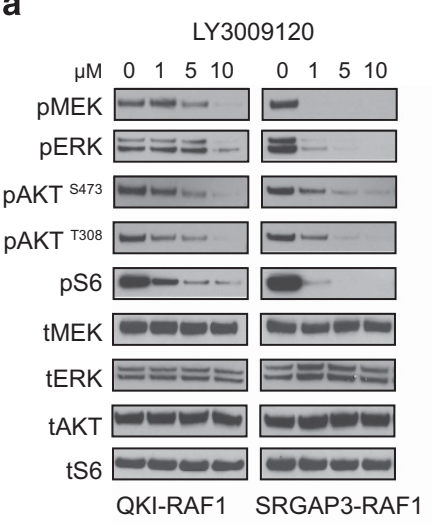

b

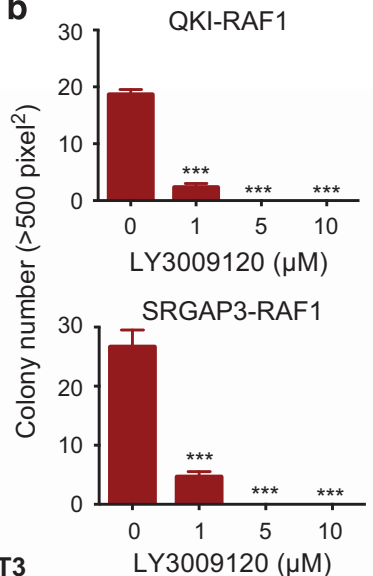

C

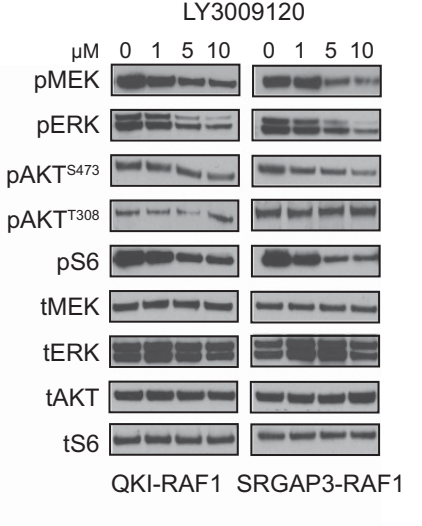

d

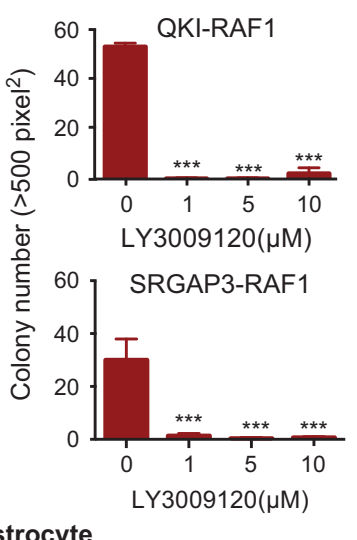

e
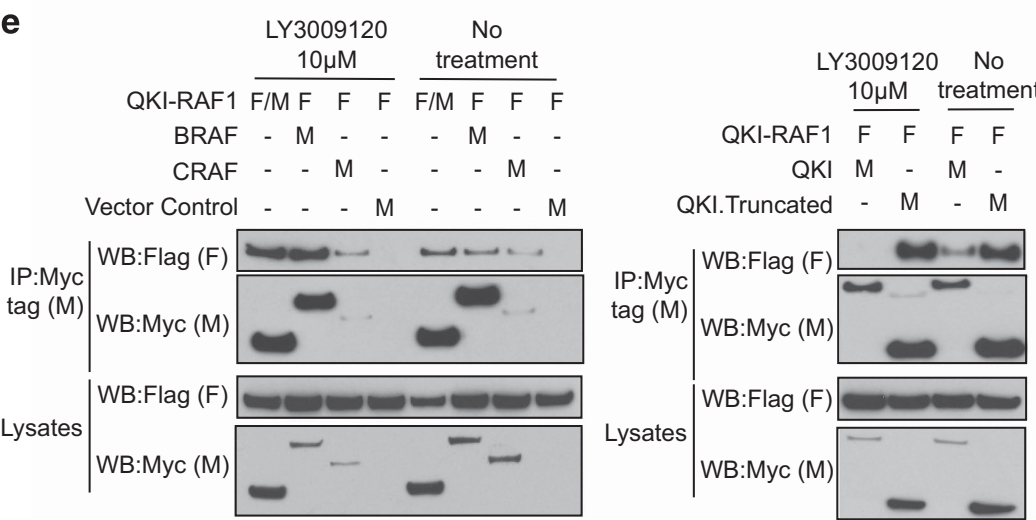

Figure 5. Novel RAF dimer inhibitor LY3009120 stabilizes and inactivates CRAF-fusion dimers. (a, c) Western blot showing effect of LY3009120 on QKI-RAF1 and SRGAP3-RAF1-mediated signaling pathways in (a) NIH3T3 cells and (c) PMAs. (b, d) Analysis of colonies in soft agar formed by CRAF-fusion expressing (b) NIH3T3 and (d) PMAs treated with LY3009120. (e) Co-IP assays showing effects of LY3009120 on QKI-RAF1 dimerization in HEK293. Error bars represent s.e.m., $n=3$. No value on bars means N.S. (non-significant), ${ }^{* * *} P$-value $<0.001$ compared with zero drug.

first report to distinguish between BRAF and CRAF/RAF1 fusions found in PAs. ${ }^{6}$ Whereas vemurafenib paradoxically activates BRAF fusions, PLX8394 suppresses KIAA1549-BRAF tumors. Contrastingly, CRAF fusions are resistant to both vemurafenib and PLX8394 but are responsive to RAF dimer inhibitor LY3009120 or co-targeting with MEKi and mTORi. These results establish distinct sensitivities of BRAF- and CRAF fusions to various inhibitors. We also identify critical contribution of the N-terminal fusion partner QKI in QKI-RAF1's resistance to existing RAFi, a finding that may be relevant to CRAF fusions in a broad category of pediatric and adult cancers.

Compared with a small fraction of PAs reported with CRAF fusions, various BRAF mutations are found more frequently including $\mathrm{BRAF}^{\mathrm{V} 600 \mathrm{E}}$ present in $6-10 \%$ of $\mathrm{PAs}$ and higher prevalence in other PLGG subtypes, ${ }^{47}$ KIAA1549-BRAF fusion found in majority of PAs and novel BRAF mutations reported by PLGG whole-genome sequencing studies. ${ }^{6}$ Despite sharing the same histopathology, our findings define distinct molecular and therapeutic profiles for CRAF-fusion and BRAF-fusion-driven tumors and suggest that targeted therapies should be tailored to the specific RAF alteration. This necessitates development of molecular stratification schemes for PLGGs as patients with similar histological diagnosis might have different molecular drivers.

Relevant patient-derived models are extremely rare for PLGGs, with no established PLGG cell lines expressing CRAF fusions. ${ }^{47-49}$ The heterologous model systems used in this study have previously been predictive of clinical response such as in the PLGG Phase II trial with sorafenib. ${ }^{29}$ These model systems indicate that CRAF fusions are oncogenic via the MAPK and PI3K/mTOR pathways. Yet, despite similar downstream signaling to BRAF fusions, CRAF fusions were found to be distinct molecular entities. Whereas KIAA1549-BRAF primarily exists as homodimers ${ }^{3}$ disrupted by PLX8394 (Figure 3C), CRAF fusions exist as RAFi-resistant homodimers and heterodimers with B/CRAF/N-terminal partner. Dependence on BRAF mutational status for RAFi sensitivity has been studied, but our findings suggest for the first time that BRAFand CRAF fusions could be distinct signaling entities based on dimerization status primarily mediated by the non-kinase fusion partner. Furthermore, other $\mathrm{N}$-terminal fusion partners of CRAF fusions, such as SRGAP3, FYCO1 and ATG7, possess dimerization domains. This raises the possibility that a common dimerizationdependent oncogenic mechanism prevails among pan-cancer CRAF fusions that needs to be established via further comprehensive testing of diverse CRAF fusions. Importantly, these mechanistic findings have significant clinical implications for patients with CRAF fusions, as clinically available RAFi would be unsuccessful in disrupting oncogenic dimers and related signaling of CRAF fusions.

LY3009120 is a pan-RAFi that has shown promise in inhibiting various NRAS, KRAS and BRAF mutations. ${ }^{45,46}$ We show LY3009120 treatment disrupting QKI-mediated interaction between QKI-RAF1 as well as stabilizing inactive homodimers and heterodimers with B/CRAF. This strongly supports our hypothesis that a RAFi can be effective against CRAF fusions if dimerization of both fusion partners is targeted, but these initial findings would require further preclinical in vivo testing.

While selumetinib is currently in clinical trials for PLGGs (ClinicalTrials.gov Identifier: NCT01089101), we have shown only 
a

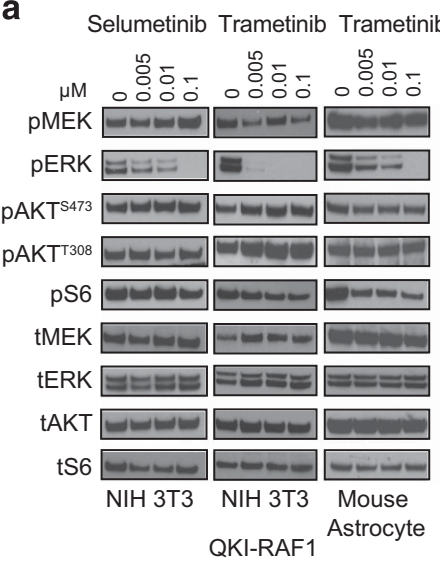

e

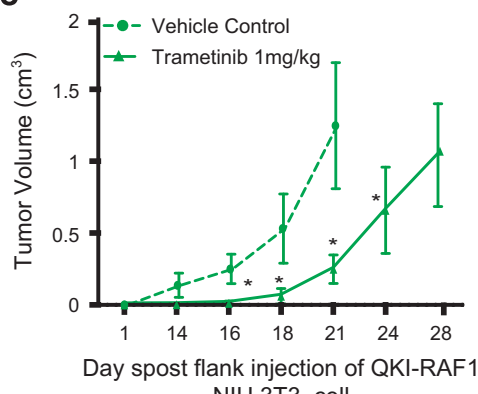

NIH 3 T3 cell b

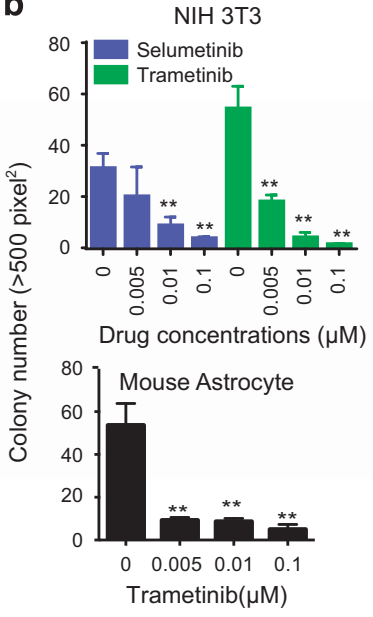

f

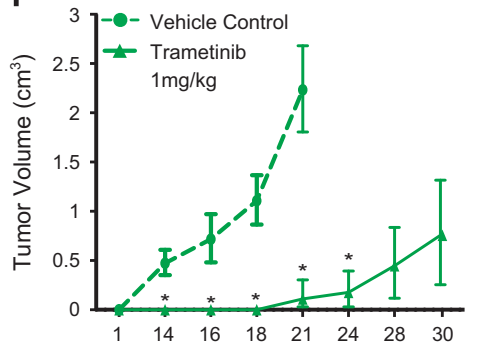

Days post flank injection of SRGAP3-RAF1

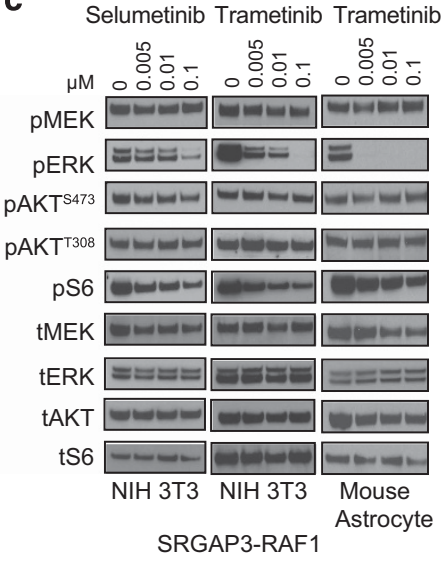

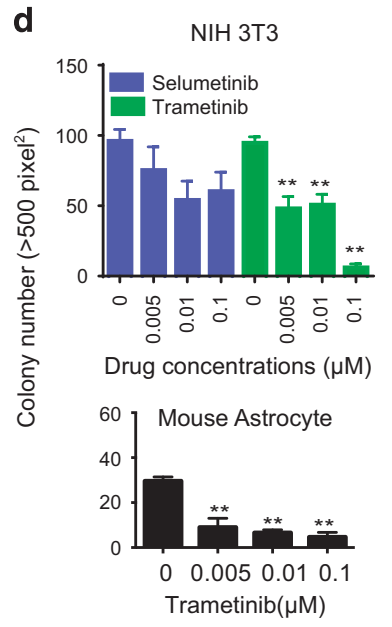

Figure 6. MEKi, Selumetinib and Trametinib, partially suppress CRAF-fusion-mediated tumor growth. (a) Western blots showing effect of Selumetinib (first panel) and Trametinib (second panel) on MAPK and PI3K/mTOR pathways in QKI-RAF1 expressing NIH3T3, and Trametinib on QKI-RAF1 expressing PMAs (third panel). (b) Effect of MEKi on QKI-RAF1-driven soft agar colony formation in NIH3T3 (top panel) and PMAs (bottom panel). (c) Western blots showing effect of Selumetinib (first panel) and Trametinib (second panel) on MAPK and PI3K/mTOR pathways in SRGAP3-RAF1 expressing NIH3T3, and Trametinib on SRGAP3-RAF1 expressing PMAs (third panel). (d) Effect of MEKi on SRGAP3RAF1-driven soft agar colony formation in NIH3T3 (top panel) and PMAs (bottom panel). Effect of MEKi on flank xenograft tumor growth in mice with stable NIH3T3-expressing (e) QKI-RAF1 and (f) SRGAP3-RAF1. Error bars represent s.e.m., $n=10$ mice. ${ }^{*} P$-value $<0.05$, ${ }^{* *} P$-value $<0.01$.

partial inhibition of CRAF fusion with selumetinib monotherapy. In BRAF-mutant cancers, selumetinib is more potent than RAFi but multi-agent therapy combining MEKi with either vemurafenib or mTORi (everolimus) has shown the most promising preclinical results based on BRAF mutational status. ${ }^{47}$ In our study, trametinib monotherapy partially suppressed CRAF-fusion-driven tumors, suggesting some oncogenic dependence on the PI3K/mTOR pathway. Combinatorial therapy using MEKi trametinib and mTORi everolimus was most effective in inhibiting the growth of QKIRAF1- and SRGAP3-RAF1-driven tumors. Safety and tolerability of combining MEKi and mTORi were tested recently in adult patients with advanced solid tumors. ${ }^{50}$ This study failed to identify a dosage that provided appropriate drug response with acceptable tolerability in adults. However, this study used a varied adult patient cohort, some of whom had previous chemotherapy and other treatments. Importantly, trametinib combined with everolimus at doses lower than what we used in this study was successful at targeting BRAF fusions (AJW, unpublished). Our preclinical results using combinatorial targeting of MAPK and $\mathrm{PI} 3 \mathrm{~K} / \mathrm{mTOR}$ pathways in CRAF fusions would suggest that this therapeutic strategy should be further explored in the pediatric setting, specifically for CRAF-fusion-harboring tumors.

Previous studies on kinase gene fusions have focused on downstream effects of truncated, activated kinases, but our study depicts a novel oncogenic role for the non-kinase fusion partner. We found that QKI-mediated dimerization of QKI-RAF1 is essential for resistance to RAFi therapy. This adds to the growing list of oncogenic roles being attributed to $\mathrm{QKI}$, including loss of expression/tumor suppressor function, altered regulation of target RNAs and maintenance of glioma stemness. ${ }^{18,51-53}$ However, the role of $\mathrm{N}$-terminal fusion partner might be fusion-specific. For example, introduction of the R401H substitution to ESRP1-RAF1, an oncogenic protein fusion that forms constitutive dimers driven by CRAF, significantly reduced MAPK signaling. ${ }^{10,54}$ Our study highlights how both genes involved in an oncogenic somatic rearrangement collaborate, predicting a vast array of potential oncogenic functions that the non-kinase partner can execute.

We have previously shown that partial loss of QKI expression in angiocentric gliomas can enhance the oncogenic profile of MYBQKI fusion. ${ }^{18}$ Because of absence of RNA-sequencing data, we could not directly verify whether there is partial loss of QKI expression in QKI-RAF1-driven tumors. While this needs further investigation, there is a possibility that monoallelic QKI loss could collaborate with the oncogenicity of QKI-RAF1. Similarly, loss of the functional SRGAP3 Rho-GTPase domains in SRGAP3-RAF1 suggests functional allelic loss of SRGAP3. This, along with contribution of SRGAP3 dimerization motifs to fusion gene oncogenicity, needs to be further explored.

The distinct subtypes of PLGGs are beginning to be associated with specific mutations such as the association of MYB-QKI with angiocentric gliomas and KIAA1549-BRAF fusions with PAs. This study adds another layer of complexity with the same histologic 


\section{a}
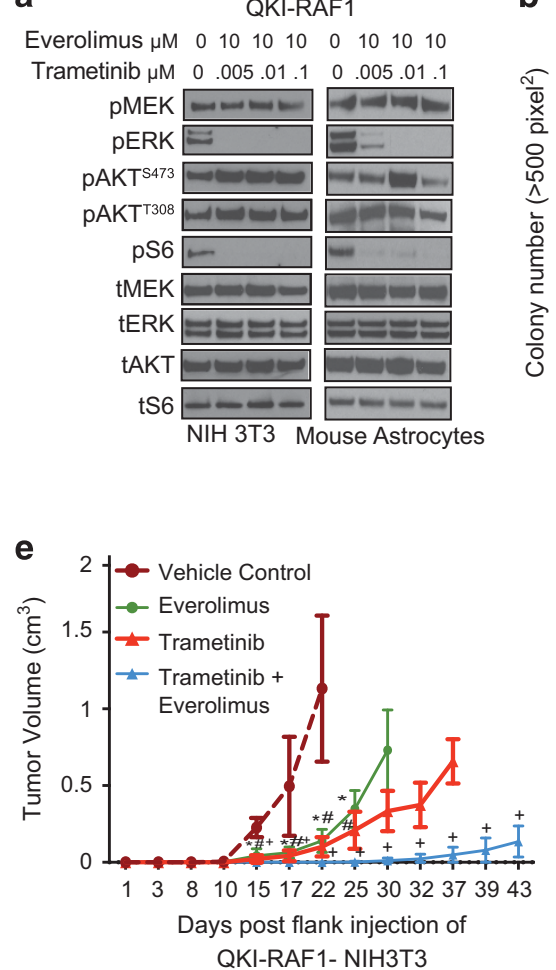

b

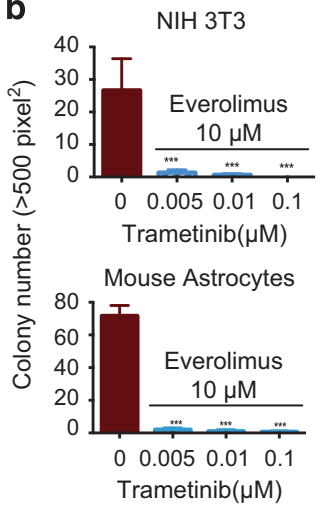

C

Everolimus $\mu \mathrm{M} \quad 0 \quad \begin{array}{llllllll}10 & 10 & 10 & 0 & 10 & 10 & 10\end{array}$

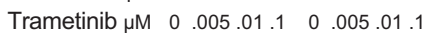

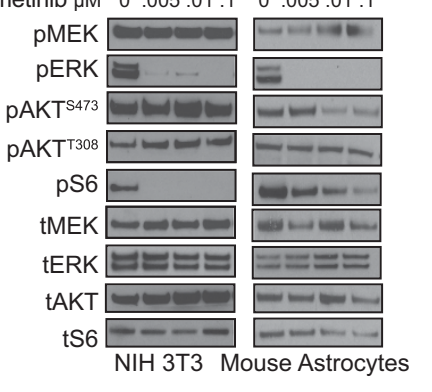

d

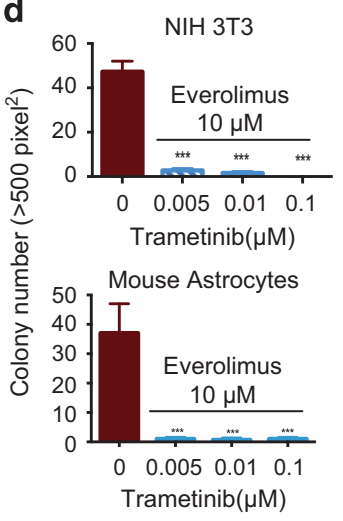

f

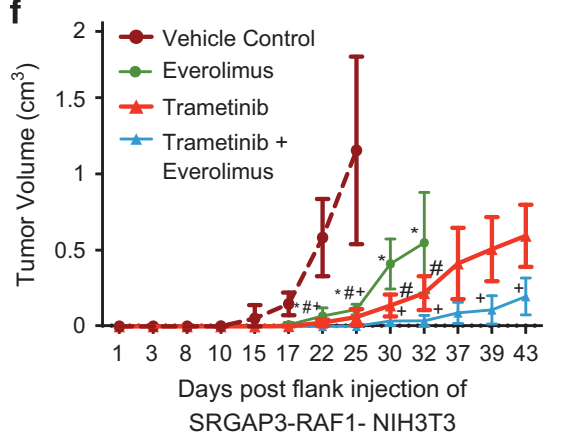

g

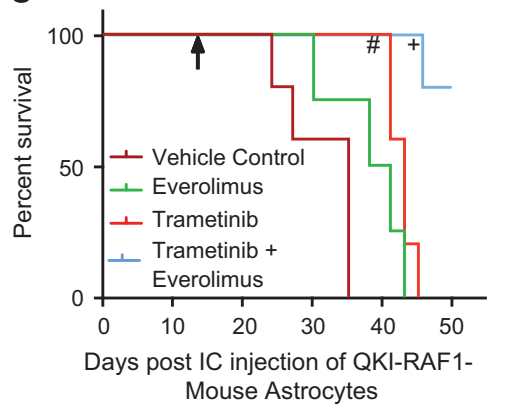

Figure 7. Combinatorial targeting of MAPK and PI3K/mTOR pathways (MEK inhibitors+mTOR inhibitors) suppresses CRAF-fusion-driven tumor growth. (a) Western blot showing effect of MEKi+mTORi on signaling pathway driven by QKI-RAF1 stably expressed in NIH3T3 (left panel) and PMAs (right panel). (b) Effect of MEKi+mTORi on QKI-RAF1-driven soft agar colony formation in NIH3T3 (top panel) and PMAs (bottom panel). (c) Western blot showing effect of MEKi+mTORi on signaling pathway driven by SRGAP3-RAF1 stably expressed in NIH3T3 (left panel) and PMAs (right panel). (d) Effect of MEKi+mTORi on SRGAP3-RAF1-driven soft agar colony formation in NIH3T3 (top panel) and PMAs (bottom panel). Error bars represent s.e.m., $n=3$. No value on bars means N.S. (non-significant), ***P-value $<0.001$ compared with zero drug. (e, $\mathbf{f}$ ) Measuring outcome of Trametinib $(1 \mathrm{mg} / \mathrm{kg})$, Everolimus $(10 \mathrm{mg} / \mathrm{kg})$ and combinatorial treatment on mouse flank xenograft growth driven by (e) QKI-RAF1 and (f) SRGAP3-RAF1 expressing NIH3T3, $n=10$ mice, plot shows s.e.m. (g) Kaplan-Meier survival plot of NSG mice orthotopically injected with PMAs overexpressing QKI-RAF1 and treated with Trametinib, Everolimus and combinatorial treatment, $n=5$ mice; arrow represents day 13 when treatments were started. Significant difference between vehicle control and respective group: *Everolimus, "Trametinib, ${ }^{+}$Combinatorial treatment.

subtype displaying different mutations and distinct therapeutic responses. Overall, we highlight the importance of molecular classification of PLGGs in assisting personalized medicine approaches, along with therapeutic implications for pan-cancer CRAF fusions.

\section{MATERIALS AND METHODS}

Cell culture

NIH3T3 and HEK293 were obtained from ATCC and maintained in $1 \times$ DMEM containing 10\% Donor Bovine Serum (DBS) and 10\% Fetal Bovine Serum (FBS), respectively. Tp53-null early-passage PMAs were kindly shared by Suzanne Baker (St Jude Children's Hospital), and maintained in DMEMF12 with $10 \%$ FBS and epidermal growth factor (EGF). ${ }^{6}$ Cell lines were routinely tested for mycoplasma infection.

Vector construction and generation of stable cell lines

SRGAP3-RAF1 and QKI-RAF1 constructs were synthesized as Gatewaycompatible entry clones. Full-length RAF1, QKI and SRGAP3 were purchased as gateway entry clones from PlasmID/Dana-Farber/Harvard Cancer Center DNA Resource Core. Subcloning was carried out to integrate SRGAP3-RAF1, QKI-RAF1, full-length QKI, RAF1 and SRGAP3 into Gateway-compatible N-MYC-tagged pMX-Puro Retroviral Vector (Cell Biolabs, San Diego, CA, USA). NIH3T3 and early-passage PMAs were transduced using infection protocol previously described. ${ }^{18}$ Gateway destination vectors with either an N-terminal MYC or FLAG tag (Invitrogen, Waltham, MA, USA) were generated for all constructs. Anti-MYC antibody (Invitrogen R951-25, 1:5000) and anti-FLAG antibody (Sigma A8592, 1:10 000 from Sigma-
Aldrich, St Louis, MO, USA) were used to detect tagged proteins along with anti-CRAF antibody (Cell Signaling \#9422 from Cell Signaling Technology, Danvers, MA, USA).

QKI-RAF1 dimerization mutants were generated by polymerase chain reaction-based site-directed mutagenesis of MYC- and FLAG-tagged constructs. RAF ${ }^{\text {R401H }}$ dimerization mutants ${ }^{35,36}$ in QKI-RAF1 were generated using primers: forward CGCAAAACACACCATGTGAACA and reverse CAGA ACAGCCACCTCATTCCT. QKI ${ }^{\mathrm{E} 48 \mathrm{G}}$ dimerization mutants ${ }^{31}$ in QKI-RAF1 were generated using primers: forward CTGGACGAAGGAATTAGCAGAG and reverse CAGCCGCTCGAGGTGGTT.

\section{Cellular drug assays}

PLX4720 and PLX8394 were provided by Plexxikon, trametinib was provided by GlaxoSmithKline and LY3009120 (Eli Lilly, Indianapolis, IN, USA) and selumetinib (Astra Zeneca, Gaithersburg, MD, USA) were purchased from Selleck Chemicals (Houston, TX, USA). These drugs were used alone or in combination with mTORi, everolimus (purchased from LC Laboratories, Woburn, MA, USA). All listed inhibitors were dissolved in dimethyl sulfoxide and stored at $-20^{\circ} \mathrm{C}$. Cells were plated at $1 \times 10^{6}$ cells/ $\mathrm{ml}$ and serum-starved, followed by exposure to indicated drug concentrations for $1 \mathrm{~h}$.

\section{Western blot analysis}

CRAF-fusion-expressing NIH3T3 and PMAs were processed for western blot analysis as described previously. ${ }^{3}$ For MAPK and PI3K/mTOR pathway analysis, pMEK (\#9154), MEK (\#4694), pERK (\#4370), ERK (\#4695), pAKT Ser473 (\#4060), pAKT Thr308 (\#4056), AKT (\#2920), pS6 (\#2215) and S6 (\#2317) antibodies from Cell Signaling were used. 
Soft-agar cellular transformation assays

Anchorage-independent growth was assessed as previously described. ${ }^{3,18}$

Co-IP assays

Interactions of SRGAP3-RAF1 and QKI-RAF1 with itself, wild-type BRAF, CRAF, QKI or SRGAP3 were assessed via co-transfections of MYC- and FLAG-tagged constructs into HEK293 using Lipofectamine 2000. HEK293 was used due to high transfection efficiency and protein expression. AntiMYC antibody-coated magnetic beads (MBL, M047-11, purchased from MBL, Woburn, MA, USA) were used to immunoprecipitate the transfected proteins from cell lysates at $4{ }^{\circ} \mathrm{C}$ overnight. Beads were washed $3 \times 15$ min at $4{ }^{\circ} \mathrm{C}$, followed by a final $1 \times$ phosphate-buffered saline wash, elution using $2 \times$ LDS (Lithium dodecyl sulfate) and heating at $70^{\circ} \mathrm{C}$ for $10 \mathrm{~min}$, and then by western blot analysis.

\section{Animal studies}

Homozygous NSG mice were obtained from Jackson Laboratories (Bar Harbor, ME, USA), bred in our animal facility and housed under aseptic conditions. We used 6-10-week-old mice with equal sex representation, randomized for treatment and no investigator blinding. The Children's Hospital of Philadelphia Institutional Animal Care and Use Committee approved all animal protocols.

Mouse flank xenograft studies with CRAF-fusion-expressing NIH3T3: $\mathrm{NIH} 3 \mathrm{T3}$ cell lines were injected subcutaneously into NSG mouse flanks ( $n=10$ each cell line, per treatment arm) and tumor growth was measured daily. Trametinib (1 mg/kg/dose) and everolimus ( $10 \mathrm{mg} / \mathrm{kg} / \mathrm{dose})$ combinatorial drug study was performed by pretreating with daily oral gavage for 1 week prior to injecting cell lines (doses chosen based on scaling from human dosing). Ellipsoid tumor volume was calculated using the formula: volume $=1 / 2\left(\right.$ length $\times$ width $\left.^{2}\right)$.

IC tumor model: $1 \times 10^{6}$ PMAs expressing SRGAP3-RAF1, QKI-RAF1 and vector control were resuspended in Matrigel basement matrix (BD Biosciences, Franklin Lakes, NJ, USA) and $2 \mu \mathrm{l}$ was injected into the right striatum of NSG mice ( $n=5 /$ cell line). Animals were monitored and killed at the onset of neurological symptoms, QKI-RAF1 on day 39 and SRGAP3RAF1 on day 42 post injection. Similar treatment doses as used in flank experiment were started on day 13 post recovery from IC injection. All animals were sacked on day 50 .

\section{UV crosslinking and co-IP assays}

We used two known RNA targets of QKI, myelin basic protein and EGR-2 ${ }^{43}$ and performed in vitro transcription and $\mathrm{P}^{32}$-radiolabeling of RNA probes. UV crosslinking with QKI or QKI-RAF1 expressing HEK293 lysates was done as described previously. ${ }^{55}$ Crosslinked protein-RNA conjugates were immuoprecipitated using anti-Myc-tagged beads and separated on an SDS-PAGE gel.

\section{Statistical analysis}

$P$-values were calculated using $t$-tests (two-sided, correction for multiple comparisons using the Holm-Sidak method). Kaplan-Meier curves and logrank (Mantel-Cox) survival analysis was used for IC model. All statistical analyses were conducted using GraphPad Prism Version 6.0 (GraphPad Software, La Jolla, CA, USA).

\section{CONFLICT OF INTEREST}

The authors declare no conflict of interest.

\section{ACKNOWLEDGEMENTS}

We thank Plexxikon Inc. for providing PLX4720 and PLX8394/PB-3 compounds and GlaxoSmithKline for providing trametinib. We greatly appreciate Jake Budlow, Tim Delaney, Namrata Choudhari and Katie Boucher for excellent technical assistance, and all members of the Resnick Lab for helpful discussion. We thank Dr Matthew Weitzman and Dr Mateusz P Koptyra for careful reading of the manuscript, and Dr Koptyra for helping with revision. This work was funded by the A Kids' Brain Tumor Cure Foundation Pediatric Low-Grade Astrocytoma Foundation (ACR), Voices Against Brain Cancer (ACR), the Children's Brain Tumor Foundation (ACR, AJW), the DamonRunyon Sohn Pediatric Fellowship Award (AJW), a Hyundai Scholar Grant (AJW), the Bear Necessities Pediatric Cancer Foundation (AJW, ACR), the Rally Foundation for Childhood Cancer Research (AJW), Thea's Star of Hope (ACR, AJW), NIH National
Center for Advancing Translational Sciences Award TL1TR000138 (HJH) and the National Institute of Health grant R01NS085336 (ACR).

\section{REFERENCES}

1 Kleihues P, Louis DN, Scheithauer BW, Rorke LB, Reifenberger G, Burger PC et al. The WHO classification of tumors of the nervous system. J Neuropathol Exp Neurol 2002; 61: 215-225, discussion 226-219.

2 Sievert AJ, Jackson EM, Gai X, Hakonarson H, Judkins AR, Resnick AC et al. Duplication of 7q34 in pediatric low-grade astrocytomas detected by high-density single-nucleotide polymorphism-based genotype arrays results in a novel BRAF fusion gene. Brain Pathol 2009; 19: 449-458.

3 Sievert AJ, Lang SS, Boucher KL, Madsen PJ, Slaunwhite E, Choudhari N et al. Paradoxical activation and RAF inhibitor resistance of BRAF protein kinase fusions characterizing pediatric astrocytomas. Proc Natl Acad Sci USA 2013; 110: 5957-5962.

4 Louis DN, Perry A, Reifenberger G, von Deimling A, Figarella-Branger D, Cavenee WK et al. The 2016 World Health Organization classification of tumors of the central nervous system: a summary. Acta Neuropathol 2016; 131: 803-820.

5 Rapp UR, Goldsborough MD, Mark GE, Bonner TI, Groffen J, Reynolds FH Jr et al. Structure and biological activity of v-raf, a unique oncogene transduced by a retrovirus. Proc Natl Acad Sci USA 1983; 80: 4218-4222.

6 Zhang J, Wu G, Miller CP, Tatevossian RG, Dalton JD, Tang B et al. Whole-genome sequencing identifies genetic alterations in pediatric low-grade gliomas. Nat Genet 2013; 45: 602-612.

7 Jones DT, Ichimura K, Liu L, Pearson DM, Plant K, Collins VP. Genomic analysis of pilocytic astrocytomas at $0.97 \mathrm{Mb}$ resolution shows an increasing tendency toward chromosomal copy number change with age. J Neuropathol Exp Neurol 2006; 65: 1049-1058.

8 Jones DT, Kocialkowski S, Liu L, Pearson DM, Ichimura K, Collins VP. Oncogenic RAF1 rearrangement and a novel BRAF mutation as alternatives to KIAA1549:BRAF fusion in activating the MAPK pathway in pilocytic astrocytoma. Oncogene 2009; 28: 2119-2123.

9 Phillips JJ, Gong H, Chen K, Joseph NM, van Ziffle J, Jin LW et al. Activating NRF1BRAF and ATG7-RAF1 fusions in anaplastic pleomorphic xanthoastrocytoma without BRAF p.V600E mutation. Acta Neuropathol 2016; 132: 757-760.

10 Palanisamy N, Ateeq B, Kalyana-Sundaram S, Pflueger D, Ramnarayanan K, Shankar $S$ et al. Rearrangements of the RAF kinase pathway in prostate cancer, gastric cancer and melanoma. Nat Med 2010; 16: 793-798.

11 Stransky N, Cerami E, Schalm S, Kim JL, Lengauer C. The landscape of kinase fusions in cancer. Nat Commun 2014; 5: 4846.

12 Yoshihara K, Wang Q, Torres-Garcia W, Zheng S, Vegesna R, Kim H et al. The landscape and therapeutic relevance of cancer-associated transcript fusions. Oncogene 2015; 34: 4845-4854.

13 Chmielecki J, Hutchinson KE, Frampton GM, Chalmers ZR, Johnson A, Shi C et al. Comprehensive genomic profiling of pancreatic acinar cell carcinomas identifies recurrent RAF fusions and frequent inactivation of DNA repair genes. Cancer Discov 2014; 4: 1398-1405.

14 Flaherty KT, Robert C, Hersey P, Nathan P, Garbe C, Milhem M et al. Improved survival with MEK inhibition in BRAF-mutated melanoma. N Engl J Med 2012; 367: 107-114.

15 Zhang C, Spevak W, Zhang Y, Burton EA, Ma Y, Habets G et al. RAF inhibitors that evade paradoxical MAPK pathway activation. Nature 2015; 526: 583-586.

16 Vernet C, Artzt K. STAR, a gene family involved in signal transduction and activation of RNA. Trends Genet 1997; 13: 479-484.

17 Friedrich VL Jr. The myelin deficit in quacking mice. Brain Res 1974; 82: 168-172.

18 Bandopadhayay P, Ramkissoon LA, Jain P, Bergthold G, Wala J, Zeid R et al. MYBQKI rearrangements in angiocentric glioma drive tumorigenicity through a tripartite mechanism. Nat Genet 2016; 48: 273-282.

$19 \mathrm{Li}$ ZZ, Kondo T, Murata T, Ebersole TA, Nishi T, Tada $\mathrm{K}$ et al. Expression of Hqk encoding a KH RNA binding protein is altered in human glioma. Jpn J Cancer Res 2002; 93: 167-177.

20 Lawrence MS, Stojanov P, Mermel CH, Robinson JT, Garraway LA, Golub TR et al. Discovery and saturation analysis of cancer genes across 21 tumour types. Nature 2014; 505: 495-501.

21 Zhao $Y$, Zhang G, Wei M, Lu X, Fu H, Feng F et al. The tumor suppressing effects of QKI-5 in prostate cancer: a novel diagnostic and prognostic protein. Cancer Biol Ther 2014; 15: 108-118.

22 Zong FY, Fu X, Wei WJ, Luo YG, Heiner M, Cao LJ et al. The RNA-binding protein QKI suppresses cancer-associated aberrant splicing. PLoS Genet 2014; 10: e1004289.

23 Bian Y, Wang L, Lu H, Yang G, Zhang Z, Fu H et al. Downregulation of tumor suppressor QKI in gastric cancer and its implication in cancer prognosis. Biochem Biophys Res Commun 2012; 422: 187-193. 
24 Carlson BR, Lloyd KE, Kruszewski A, Kim IH, Rodriguiz RM, Heindel C et al. WRP/srGAP3 facilitates the initiation of spine development by an inverse F-BAR domain, and its loss impairs long-term memory. J Neurosci 2011; 31: 2447-2460.

25 Lahoz A, Hall A. A tumor suppressor role for srGAP3 in mammary epithelial cells. Oncogene 2013; 32: 4854-4860.

26 Heidecker G, Huleihel M, Cleveland JL, Kolch W, Beck TW, Lloyd P et al. Mutational activation of c-raf- 1 and definition of the minimal transforming sequence. Mol Cell Biol 1990; 10: 2503-2512.

27 Stanton Jr VP, Nichols DW, Laudano AP, Cooper GM. Definition of the human raf amino-terminal regulatory region by deletion mutagenesis. Mol Cell Biol 1989; 9: 639-647.

28 Henne WM, Kent HM, Ford MG, Hegde BG, Daumke O, Butler PJ et al. Structure and analysis of FCHo2 F-BAR domain: a dimerizing and membrane recruitment module that effects membrane curvature. Structure 2007; 15: 839-852.

29 Karajannis MA, Legault G, Fisher MJ, Milla SS, Cohen KJ, Wisoff JH et al. Phase II study of sorafenib in children with recurrent or progressive low-grade astrocytomas. Neuro Oncol 2014; 16: 1408-1416.

30 Rajakulendran T, Sahmi M, Lefrancois M, Sicheri F, Therrien M. A dimerizationdependent mechanism drives RAF catalytic activation. Nature 2009; 461: 542-545.

31 Chen T, Richard S. Structure-function analysis of Qk1: a lethal point mutation in mouse quaking prevents homodimerization. Mol Cell Biol 1998; 18: 4863-4871.

32 Ali M, Broadhurst RW. Solution structure of the QUA1 dimerization domain of pXqua, the Xenopus ortholog of Quaking. PLoS One 2013; 8: e57345.

33 Hatzivassiliou G, Song K, Yen I, Brandhuber BJ, Anderson DJ, Alvarado R et al. RAF inhibitors prime wild-type RAF to activate the MAPK pathway and enhance growth. Nature 2010; 464: 431-435.

34 Bollag G, Hirth P, Tsai J, Zhang J, Ibrahim PN, Cho H et al. Clinical efficacy of a RAF inhibitor needs broad target blockade in BRAF-mutant melanoma. Nature 2010; 467: 596-599.

35 Freeman AK, Ritt DA, Morrison DK. The importance of Raf dimerization in cell signaling. Small GTPases 2013; 4: 180-185.

36 Freeman AK, Ritt DA, Morrison DK. Effects of Raf dimerization and its inhibition on normal and disease-associated Raf signaling. Mol Cell 2013; 49: 751-758.

37 Poulikakos PI, Zhang C, Bollag G, Shokat KM, Rosen N. RAF inhibitors transactivate RAF dimers and ERK signalling in cells with wild-type BRAF. Nature 2010; 464: 427-430.

$38 \mathrm{Wu}$ X, Yin J, Simpson J, Kim KH, Gu S, Hong JH et al. Increased BRAF heterodimerization is the common pathogenic mechanism for noonan syndromeassociated RAF1 mutants. Mol Cell Biol 2012; 32: 3872-3890.

39 Antony R, Emery CM, Sawyer AM, Garraway LA. C-RAF mutations confer resistance to RAF inhibitors. Cancer Res 2013; 73: 4840-4851.

40 Varga A, Ehrenreiter K, Aschenbrenner B, Kocieniewski P, Kochanczyk M, Lipniacki $T$ et al. RAF1/BRAF dimerization integrates the signal from RAS to ERK and ROKalpha. Sci Signal 2017; 10: pii: eaai8482.

41 Beuck C, Qu S, Fagg WS, Ares Jr M, Williamson JR. Structural analysis of the quaking homodimerization interface. J Mol Biol 2012; 423: 766-781.

42 Chen AJ, Paik JH, Zhang H, Shukla SA, Mortensen R, Hu J et al. STAR RNA-binding protein Quaking suppresses cancer via stabilization of specific miRNA. Genes Dev 2012; 26: 1459-1472.

43 Galarneau A, Richard S. Target RNA motif and target mRNAs of the Quaking STAR protein. Nat Struct Mol Biol 2005; 12: 691-698.
44 Henry JR, Kaufman MD, Peng SB, Ahn YM, Caldwell TM, Vogeti L et al. Discovery of 1-(3,3-dimethylbutyl)-3-(2-fluoro-4-methyl-5-(7-methyl-2-(methylamino)pyrido [2,3- d]pyrimidin-6-yl)phenyl)urea (LY3009120) as a pan-RAF inhibitor with minimal paradoxical activation and activity against BRAF or RAS mutant tumor cells. J Med Chem 2015; 58: 4165-4179.

45 Peng SB, Henry JR, Kaufman MD, Lu WP, Smith BD, Vogeti S et al. Inhibition of RAF isoforms and active dimers by LY3009120 leads to anti-tumor activities in RAS or BRAF mutant cancers. Cancer Cell 2015; 28: 384-398.

46 Chen SH, Zhang Y, Van Horn RD, Yin T, Buchanan S, Yadav V et al. Oncogenic BRAF deletions that function as homodimers and are sensitive to inhibition by RAF dimer inhibitor LY3009120. Cancer Discov 2016; 6: 300-315.

47 Olow A, Mueller S, Yang X, Hashizume R, Meyerowitz J, Weiss WA et al. BRAF status in personalizing treatment approaches for pediatric gliomas. Clin Cancer Res 2016; 22: 5312-5321.

48 Bax DA, Little SE, Gaspar N, Perryman L, Marshall L, Viana-Pereira M et al. Molecular and phenotypic characterisation of paediatric glioma cell lines as models for preclinical drug development. PLoS One 2009; 4: e5209.

49 Selt F, Hohloch J, Hielscher T, Sahm F, Capper D, Korshunov A et al. Establishment and application of a novel patient-derived KIAA1549:BRAF-driven pediatric pilocytic astrocytoma model for preclinical drug testing. Oncotarget 2017; 8: 11460-11479.

50 Tolcher AW, Bendell JC, Papadopoulos KP, Burris HA 3rd, Patnaik A, Jones SF et al. A phase IB trial of the oral MEK inhibitor trametinib (GSK1120212) in combination with everolimus in patients with advanced solid tumors. Ann Oncol 2015; 26: 58-64.

51 Danan-Gotthold M, Golan-Gerstl R, Eisenberg E, Meir K, Karni R, Levanon EY. Identification of recurrent regulated alternative splicing events across human solid tumors. Nucleic Acids Res 2015; 43: 5130-5144.

52 Conn SJ, Pillman KA, Toubia J, Conn VM, Salmanidis M, Phillips CA et al. The RNA binding protein quaking regulates formation of circRNAs. Cell 2015; 160: 1125-1134.

53 Shingu T, Ho AL, Yuan L, Zhou X, Dai C, Zheng S et al. Qki deficiency maintains stemness of glioma stem cells in suboptimal environment by downregulating endolysosomal degradation. Nat Genet 2017; 49: 75-86.

54 Yao Z, Torres NM, Tao A, Gao Y, Luo L, Li Q et al. BRAF mutants evade ERKdependent feedback by different mechanisms that determine their sensitivity to pharmacologic inhibition. Cancer Cell 2015; 28: 370-383.

55 Rothrock CR, House AE, Lynch KW. HnRNP L represses exon splicing via a regulated exonic splicing silencer. EMBO J 2005; 24: 2792-2802.

(c) (i) $\Theta$ This work is licensed under a Creative Commons Attributioncc) NonCommercial-NoDerivs 4.0 International License. The images or other third party material in this article are included in the article's Creative Commons license, unless indicated otherwise in the credit line; if the material is not included under the Creative Commons license, users will need to obtain permission from the license holder to reproduce the material. To view a copy of this license, visit http:// creativecommons.org/licenses/by-nc-nd/4.0/

(c) The Author(s) 2017

Supplementary Information accompanies this paper on the Oncogene website (http://www.nature.com/onc) 